Prepared for the U.S. Department of Energy

under Contract DE-AC05-76RL01830

\title{
Enrichment Assay Methods Development for the Integrated Cylinder Verification System
}

\section{LE Smith}

AC Misner

BK Hatchell

MM Curtis

October 2009

\section{Pacific Northwest}

NATIONAL LABORATORY

Proudly Operated by Battelle Since 1965 


\title{
DISCLAIMER
}

This report was prepared as an account of work sponsored by an agency of the United States Government. Neither the United States Government nor any agency thereof, nor Battelle Memorial Institute, nor any of their employees, makes any warranty, express or implied, or assumes any legal liability or responsibility for the accuracy, completeness, or usefulness of any information, apparatus, product, or process disclosed, or represents that its use would not infringe privately owned rights. Reference herein to any specific commercial product, process, or service by trade name, trademark, manufacturer, or otherwise does not necessarily constitute or imply its endorsement, recommendation, or favoring by the United States Government or any agency thereof, or Battelle Memorial Institute. The views and opinions of authors expressed herein do not necessarily state or reflect those of the United States Government or any agency thereof.

\author{
PACIFIC NORTHWEST NATIONAL LABORATORY \\ operated by \\ BATTELLE \\ for the \\ UNITED STATES DEPARTMENT OF ENERGY \\ under Contract DE-AC05-76RL01830
}

Printed in the United States of America

Available to DOE and DOE contractors from the

Office of Scientific and Technical Information,

P.O. Box 62, Oak Ridge, TN 37831-0062;

ph: (865) 576-8401

fax: $(865) 576-5728$

email: reports@adonis.osti.gov

\footnotetext{
Available to the public from the National Technical Information Service, U.S. Department of Commerce, 5285 Port Royal Rd., Springfield, VA 22161 ph: (800) 553-6847 fax: $(703) 605-6900$

email: orders@ntis.fedworld.gov

online ordering: http://www.ntis.gov/ordering.htm
}

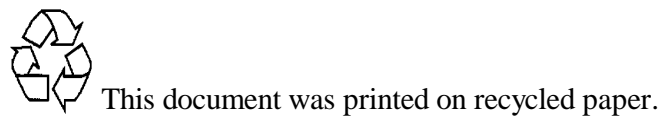




\title{
Enrichment Assay Methods Development for the Integrated Cylinder Verification System
}

\author{
LE Smith \\ AC Misner \\ BK Hatchell \\ MM Curtis
}

October 2009

Prepared for

the U.S. Department of Energy

under Contract DE-AC05-76RL01830

Pacific Northwest National Laboratory

Richland, Washington 99352 



\section{Summary}

International Atomic Energy Agency (IAEA) inspectors currently perform periodic inspections at uranium enrichment plants to verify $\mathrm{UF}_{6}$ cylinder enrichment declarations. Measurements are typically performed with handheld high-resolution instruments on a statistically determined sampling of cylinders taken to be representative of the facility's entire product-cylinder inventory. Pacific Northwest National Laboratory (PNNL) is developing a concept to automate the verification of enrichment plant cylinders to enable 100 percent product-cylinder verification and potentially, mass-balance calculations on the facility as a whole (by also measuring feed and tails cylinders). The Integrated Cylinder Verification System (ICVS) could be located at key measurement points to positively identify each cylinder, measure its mass and enrichment, store the collected data in a secure database (mailbox), and maintain continuity of knowledge on measured cylinders until IAEA inspector arrival.

PNNL's ICVS concept uses measurement systems that can be operated in an unattended mode: medium-resolution scintillators for gamma-ray spectroscopy and moderated He-3 neutron detectors. Prior proof-of-principle studies of advanced nondestructive assay (NDA) methods for automated enrichment assay, particularly neutron-related signatures spawned by U-234 alpha emission, have encouraged further development of NDA methods suitable for an ICVS prototype. The work described in this report was supported by NA-243 funding in late FY09, following completion of the initial PNNL Laboratory Directed Research and Development (LDRD)-funded study that focused on non-traditional NDA signatures. A proposal has been submitted to NA-241 for design and testing of a prototype ICVS that utilizes both non-traditional and traditional NDA signatures. Work on the components of the ICVS that do not involve research and development will continue to be supported under NA-243, and are addressed in separate reports.

The three main objectives of this FY09 project are summarized here and described in more detail in the report: 1) Develop a preliminary design for a prototype NDA system, 2) Refine PNNL's MCNP models of the NDA system, and 3) Procure and test key pulse-processing components. Progress against these tasks to date, and next steps, are discussed. 



\section{Acknowledgments}

The authors express their appreciation to Dunbar Lockwood of the National Nuclear Security Administration's Office of International Regimes and Agreements (NA-243) for his support of this work. We would also like to thank Carrie Mathews of Pacific Northwest National Laboratory for her coordination and guidance of this task, which is a part of a collection of concepts funded by NA-243 for the improvement of enrichment plants safeguards. Finally, we thank Jon Phillips and Hal Undem of the Pacific Northwest National Laboratory Sustainable Nuclear Power Initiative for financial support of prior technical work on nondestructive assay methods for automated cylinder verification. 



\section{Acronyms and Abbreviations}

ADC

IAEA

ICVS

LDRD

NDA

PNNL analog-to-digital converter

International Atomic Energy Agency

Integrated Cylinder Verification System

Laboratory Directed Research and Development

nondestructive assay

Pacific Northwest National Laboratory 



\section{Contents}

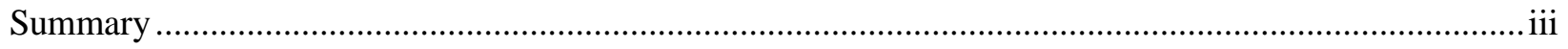

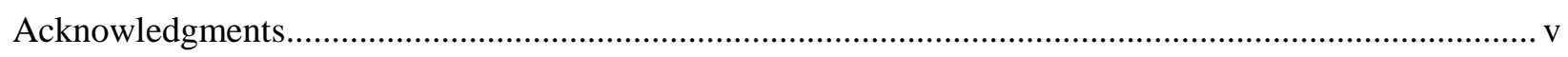

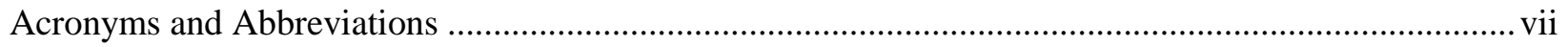

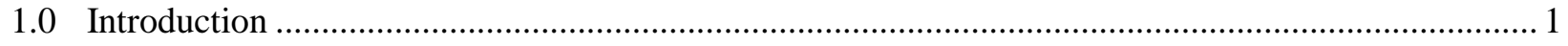

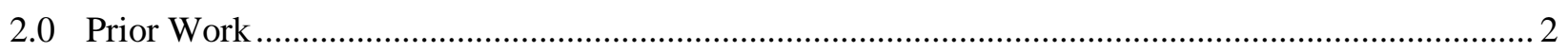

3.0 Scope and Results for Current NA-243 Project............................................................................ 3

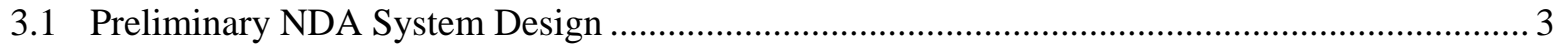

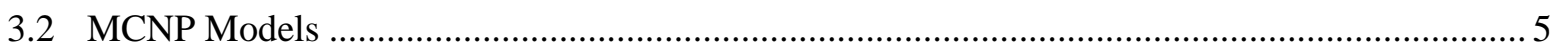

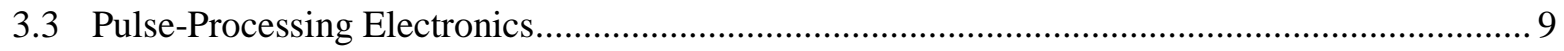

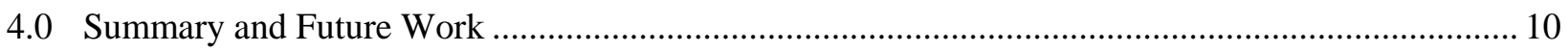

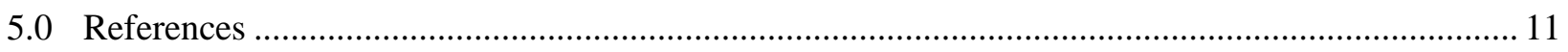

Appendix A: Summary of FY10-12 Proposal .................................................................................... A.1 


\section{Figures}

1 Photograph of experimental setup at AREVA fuel fabrication facility during April 2009 measurements....

2 Conceptual overview of the prototype NDA system that can collect both traditional 185-keV signatures and the non-traditional signatures that can support full-volume assay and automated wall-thickness correction.

3 Top: Collimated gamma-ray spectrometer module that allows translation of the sensor in the surrounding shielding to adjust the field of view, and a collimating plug in the end to further reduce the low-energy field of view. Bottom: Early concept of the positioning system that will be used to align the gamma-ray spectrometer array and He-3 neutron detectors to a 30B cylinder

4 Cross section through MCNP model used to support enrichment assay methods development 6

5 Examples of MCNP-modeled responses for the $7.5 \mathrm{~cm} \times 7.5 \mathrm{~cm} \mathrm{NaI}(\mathrm{Tl})$ and $\mathrm{LaBr}_{3}(\mathrm{Ce})$ spectrometers depicted in Figure 4 


\subsection{Introduction}

International Atomic Energy Agency (IAEA) inspectors currently perform periodic inspections at uranium enrichment plants to verify $\mathrm{UF}_{6}$ cylinder enrichment declarations. Measurements are typically performed with handheld high-resolution sensors on a sampling of cylinders taken to be representative of the facility's entire product-cylinder inventory. As additional enrichment plants come online to support the expansion of nuclear power, reducing person-days of inspection time will take on greater importance. Pacific Northwest National Laboratory (PNNL) is developing a concept to automate the verification of enrichment plant cylinders to enable 100 percent product-cylinder verification and potentially, massbalance calculations on the facility as a whole (by also measuring feed and tails cylinders). The Integrated Cylinder Verification System (ICVS) could be located at key measurement points to positively identify each cylinder, measure its mass and enrichment, store the collected data in a secure database, and maintain continuity of knowledge on measured cylinders until IAEA inspector arrival (Curtis et al. 2009). Given the potential for reduced inspector presence, the operational and manpower-reduction benefits of the portal concept are significant. The key technical question is whether the cylinder portal concept can meet, or potentially improve upon, the enrichment verification performance of today's technologies.

PNNL's ICVS concept uses instruments that can be operated in an unattended mode: mediumresolution scintillators (e.g., $\mathrm{NaI}(\mathrm{Tl})$ ) for gamma-ray spectroscopy and moderated He-3 neutron detectors. While the medium-resolution scintillators are a sacrifice in energy resolution, they do provide improved collection efficiency for signatures above $1 \mathrm{MeV}$. When compared to today's enrichment meter technologies that use only the weakly penetrating 185-keV line, the ICVS sensor combination allows the exploitation of additional, more-penetrating signatures: neutrons produced from F-19( $\alpha, n)$ reactions (spawned primarily from U-234 alpha emission) and high-energy gamma rays (extending up to $8 \mathrm{MeV}$ ) induced by neutrons interacting in the steel cylinder. These indirect measures of U-235 require a relatively stable U-234/U-235 ratio in the product material to be useful for enrichment verification purposes (Smith et al. 2009).

The hypothesis in ICVS development is that the U-234/U-235 ratio is sufficiently constant at the specific facility where the automated system is installed to use neutron-related signatures as an indirect measure of U-235 enrichment. Further, these "non-traditional” highly penetrating signatures can be combined with a modified form of "traditional" $185-\mathrm{keV}$ enrichment measurements to meet target uncertainties for the verification of cylinders. If successful, this new enrichment assay approach would provide two important benefits over today's handheld enrichment-meter method:

1. Automated operation to enable 100 percent verification of cylinders entering and leaving the facility (feed, product, and tails), and a significant reduction in IAEA manpower needed to achieve that facility mass balance;

2. Assay of the entire cylinder volume (rather than a small fraction of that volume) for accurate enrichment verification of non-homogenized cylinders (e.g., tails) and potential diversion scenarios (e.g., material substitution). 


\subsection{Prior Work}

In prior work funded by PNNL Laboratory Directed Research and Development (LDRD), PNNL studied the "non-traditional" enrichment assay aspects of the ICVS concept: neutron and high-energy gamma-ray signatures, the radiation sensors designed to collect those signatures, and proof-of-principle cylinder measurements and analysis. Results from this study, which included measurements of Type 30B cylinders at an AREVA fuel fabrication plant in Richland, Washington (see Figure 1) indicated that these non-traditional signatures have the potential to meet target uncertainty values for 30B product cylinders (5\%; Kuhn 2001), even when the traditional assay signatures are not used (Smith et al. 2009).

Preliminary analyses also indicated that if the non-traditional signatures are combined with the traditional 185-keV assay method, even lower uncertainties can be achieved and other important anomaly-detection features could be realized (e.g., ratio of U-234/U-235 or U-232/U-235).

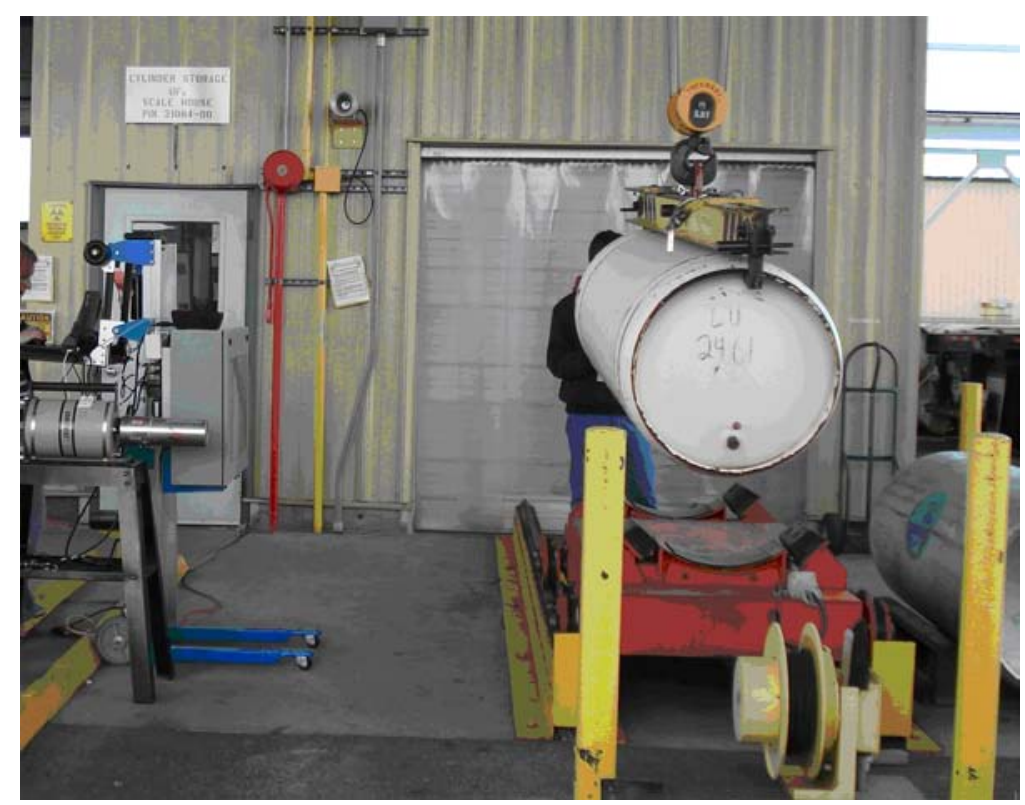

Figure 1. Photograph of experimental setup at AREVA fuel fabrication facility during April 2009 measurements. The proof-of-principle NaI/He-3 panel and the $120 \%$ HPGe are at left, approximately $1.5 \mathrm{~m}$ from the cylinder wall. At right, a cylinder is being lowered onto the cylinder trolley.

PNNL's prior work in nondestructive assay (NDA) methods development has encouraged further study on the concept of a cylinder verification station that performs accurate unattended enrichment assay of all cylinders entering (feed) and leaving (product and tail) an enrichment facility, thereby enabling verification of the facility's U-235 mass balance, and at the same time reducing IAEA manpower requirements for achieving that verification. 


\subsection{Scope and Results for Current NA-243 Project}

The FY09 PNNL LDRD results described above led to further study of the ICVS concept under NA-243 funding in late FY09. It is the enrichment-assay portion of that NA-243 scope that is the subject of this report, while non-NDA components (e.g., surveillance and mailbox concepts) are described in companion PNNL reports. There were three main objectives of the enrichment assay scope described here:

1. Develop a preliminary design for a prototype NDA system that can be used in FY10 measurements at AREVA and FY11 measurements at an operating gas centrifuge enrichment plant;

2. Refine PNNL's MCNP models of the NDA system to enable the accurate simulation of gammaray spectrometer and neutron sensor responses, in support of design optimization and algorithm development; and

3. Procure and test a few pulse-processing components that, when combined with existing PNNL instrumentation, will provide the hardware backbone of the prototype NDA system.

These objectives and progress on each during this project (June 2009 to October 2009) are described in the next sections.

\subsection{Preliminary NDA System Design}

PNNL's previous proof-of-principle measurements at AREVA and subsequent data analysis supported a number of findings important to the design of a prototype NDA system suitable for the next phase of ICVS development.

- Gamma-ray spectrometer types and sizes: The high-energy gamma-ray signatures that derive from neutron emissions are intense enough that modest-sized, medium-resolution scintillators with high atomic number and density (e.g., a $7.5 \mathrm{~cm} \times 7.5 \mathrm{~cm} \mathrm{NaI(Tl)} \mathrm{spectrometer)} \mathrm{can} \mathrm{be} \mathrm{used} \mathrm{for} \mathrm{collection.}$ This means that standard (cost-effective) commercially available sensors can be used, but the pulseprocessing systems must be designed to collect an unusually wide range of energies ( $25 \mathrm{keV}$ to over $8,000 \mathrm{keV}$, compared to a more conventional $25 \mathrm{keV}$ to 1,500 keV). Based on these results, PNNL intends to use an array of $\mathrm{NaI}(\mathrm{Tl})$ and $\mathrm{LaBr}_{3}(\mathrm{Ce})$ scintillators in combination with digital signal processing components that provide at least 8,000 channels between 25 and 8,000 keV of deposited energy.

- Gamma-ray spectrometer array design: Certain combinations of gamma rays emitted by the cylinder have the potential to support automated wall-thickness corrections that could perform the same function that handheld ultrasonic gauges do in today's IAEA cylinder verification approach. If successfully developed, this novel NDA technique could substantially improve the accuracy of automated traditional 185-keV assay using medium-resolution scintillators, and thereby close the gap in achievable uncertainty between the ICVS unattended assay and today's assay using cryogenically cooled high-resolution handhelds. In keeping with the goal of full-volume cylinder assay, an array of four collimated gamma-ray spectrometers along the length of the cylinder will provide multiple, "volume-averaged" estimates of enrichment and wall thickness. This ICVS design feature should 
provide a considerable advantage over the very localized assay performed today with handheld instruments, particularly for cylinders that are not homogeneous (e.g., tail cylinders or intentional material substitutions).

- Gamma-ray spectrometer collimation adaptability: The count rates expected in modest-sized, medium-resolution scintillators positioned very close to the cylinder wall may overwhelm standard pulse-processing electronics, which favors the use of a highly collimated detector array. However, the collection of the high-energy (but less intense) signatures favors a wider field of view, and therefore less collimation. The appropriate balance can be preliminarily gauged through simulation, but actual cylinder measurements are needed to support a final design determination. Consequently, the prototype design should provide significant adaptability in the collimation approach. In the preliminary design, a spectrometer translates through the surrounding lead shield to adjust the sensor's field of view. A plug on the end of the detector shield further collimates high-yield but lowenergy gamma rays (e.g., $185 \mathrm{keV}$ ) using a pinhole aperture, while allowing the majority of loweryield but higher-energy gamma rays to enter the detector.

- Platform adaptability, cost-effectiveness, low-impact form factor: The design of the NDA prototype should have sufficient flexibility that various NDA optimization ideas can be tested, yet the prototype should be easy to transport and move through the facilities where it might be tested (e.g., AREVA and the National Enrichment Facility). Both requirements tend designers toward modularity and simplicity. To reduce the cost and development time of the prototype NDA system, PNNL design engineers have queried industry to use, as much as possible, off-the-shelf shielding and support components. For example, a commercially available detector collimator will be adapted for the gamma-ray spectrometer array to facilitate adjustments to the collimation and aperture. The gammaray spectrometer array and He-3 neutron detectors will be mounted to a commercial scissor lift table for coarse positioning. Additional bearings and actuators will accommodate precise adjustments to height, stand-off distance, viewing angle, and longitudinal location.

A conceptual overview of the NDA system is given in Figure 2. This concept reflects the design ideas described above.

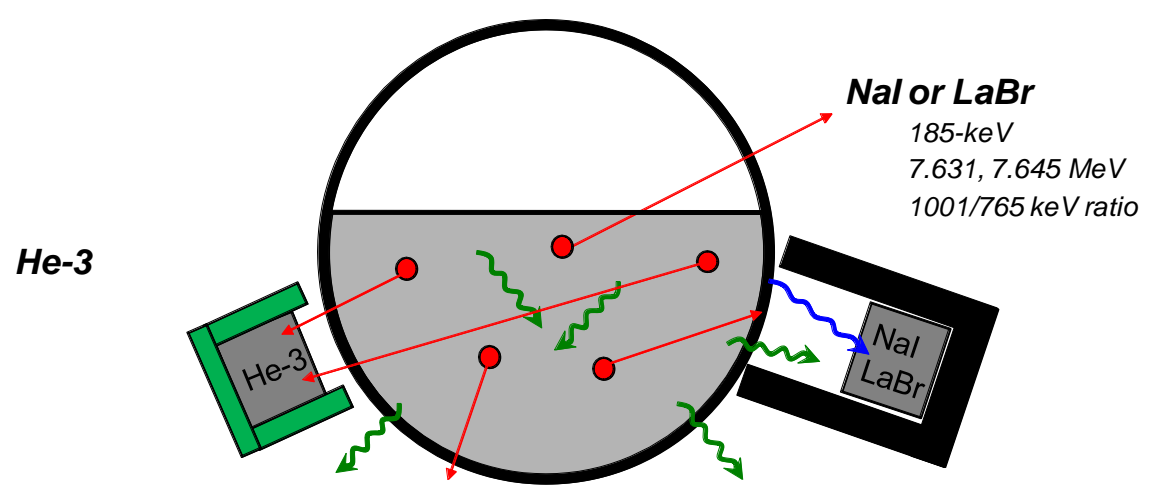

Figure 2. Conceptual overview of the prototype NDA system that can collect both traditional 185-keV signatures (using collimated medium-resolution scintillators such as $\mathrm{NaI}\left(\mathrm{Tl}\right.$ ) or $\mathrm{LaBr}_{3}$ ) and the nontraditional signatures that can support full-volume assay (e.g., 7.6-MeV neutron-induced lines) and automated wall-thickness correction. He-3-based neutron detection is depicted, but may not be necessary in an ICVS prototype because the high-energy gamma-ray signature is a corollary for the neutron emissions. 
A schematic overview of the preliminary NDA prototype design is shown in Figure 3. This early design concept will be the foundation of the final design and fabrication work of the full development project in FY10, should it be funded by NA-241.

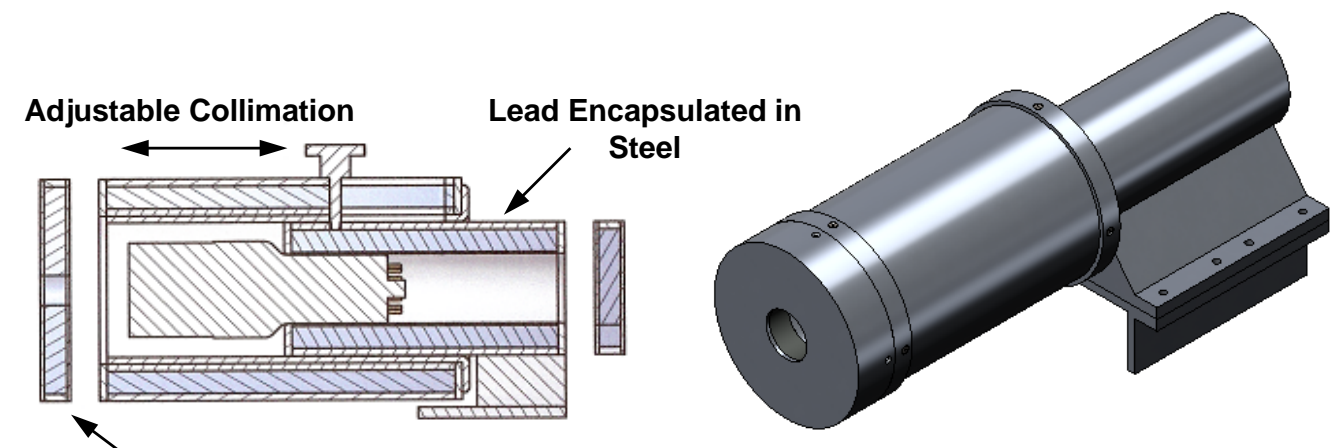

Adaptable Shielding Aperture

\section{Detector/Collimator Mounted to Linear Bearing}
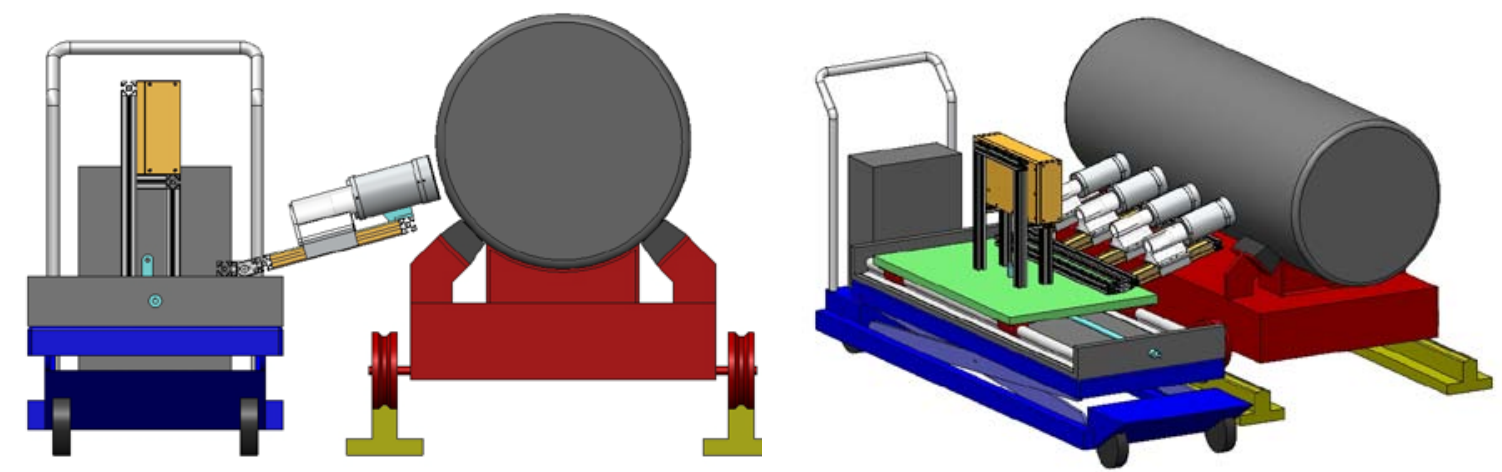

Figure 3. Top: Collimated gamma-ray spectrometer module that allows translation of the sensor in the surrounding shielding to adjust the field of view, and a collimating plug in the end to further reduce the low-energy field of view. Bottom: Early concept of the positioning system that will be used to align the gamma-ray spectrometer array and He-3 neutron detectors to a 30B cylinder. Mechanical actuators will accommodate vertical, longitudinal, and angular adjustability.

\subsection{MCNP Models}

High-fidelity modeling of the $\mathrm{UF}_{6}$ cylinder assay scenario is a time- and resource-efficient complement to other development tasks. For example, the impact of different background shielding configurations can be quickly explored in simulation, but is time-consuming and expensive in the field. Simulation can also be used to generate large libraries of "virtual assay signatures" to support algorithm development, over the parameter space important to a viable instrument (e.g., enrichment levels, U-232 contamination levels, and wall-thickness variation). PNNL developed a functional and preliminarily validated modeling framework in previous LDRD work, during an NA-243-supported Safeguards Student Internship (Matthew Gidden, graduate student at University of Wisconsin). 
In this current scope, an NA-243-supported PNNL post-doctoral researcher (Alex Misner from Oregon State University) is refining these MCNP models to be more realistic to the AREVA measurement configuration, more computationally efficient (i.e., variance reduction techniques are being added), and to reflect the latest in prototype design choices (e.g., collimator design, array arrangement). A slice through the MCNP model, showing both the proof-of-principle instrumentation panel used in April 2009 measurements at AREVA (at left, approximately $150 \mathrm{~cm}$ from the cylinder) and the preliminary prototype design (close to the cylinder wall) is displayed in Figure 4.

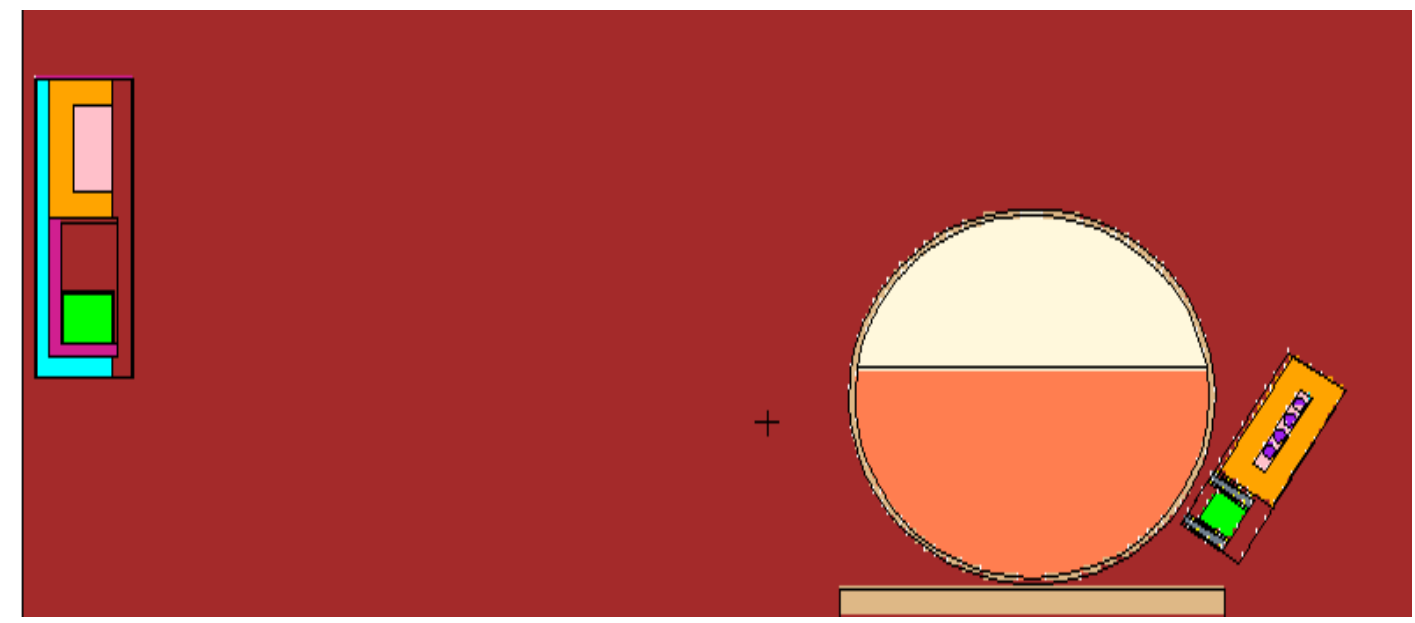

Figure 4. Cross section through MCNP model used to support enrichment assay methods development. The 30B cylinder is viewed by PNNL's proof-of-principle instrumentation panel (at far left, used during April 2009 AREVA measurements) and an array of collimated medium-resolution spectrometers with neutron module (on right, preliminary design of prototype intended for use in FY10 measurements).

Once the physical model of the enrichment portal monitor has been assembled, the next step is to model the various source terms contributing to the measured signature in each measurement scenario. The primary photon sources considered in this modeling include:

- Characteristic gamma rays from the prominent gamma-ray emitting uranium isotopes (e.g., U-232, U-235, and U-238) and their daughters, assuming the decay time since chemical processing ranges up to 6 months (a range defined by secular equilibrium between U-238 and its decay product Pa-234m, which occurs about six months following chemical separation);

- Neutron-induced gamma rays, including capture and inelastic scattering (e.g., Fe (n, $\gamma$ ) reaction in the cylinder wall);

- Spontaneous fission and induced fission gamma rays; and

- Gamma decay of ${ }^{22} \mathrm{Na}(1.275 \mathrm{MeV})$ created from ${ }^{19} \mathrm{~F}(\alpha, \mathrm{n}){ }^{22} \mathrm{Na}$ reactions.

In the case of neutrons, only the total number collected per unit time is recorded (no coincidence or energy spectroscopy). Neutron signatures to model include:

- Prompt neutrons from spontaneous fission,

- Prompt neutrons from induced fission, 
- Delayed neutrons from spontaneous and induced fission, and

- Neutrons from ${ }^{19} \mathrm{~F}(\alpha, \mathrm{n}){ }^{22} \mathrm{Na}$ reactions.

Once the source terms and geometry have been defined, radiation transport methods are used to predict how the various fields will be attenuated and collected by the portal sensors (i.e., NaI spectrometers and neutron counters). Because this is a high-attenuation, high-scattering, high-dose-rate environment, analog Monte Carlo transport methods can be extremely time-consuming (hours or days) for a single cylinder source term or parameter (e.g., wall thickness) variant. Consequently, running the numerous (tens to hundreds) scenarios needed to capture the systematic variations in a realistic environment becomes difficult. To accelerate the modeling and support a flexible and diverse parameter study, PNNL is developing variance reduction techniques that can significantly reduce computational times for cylinder simulations. Those techniques involve the use of a deterministically calculated adjoint flux solution as an importance mapping for forward MCNP calculations. Some of this work is being done in collaboration with Oak Ridge National Laboratory (Mosher et al. 2009) and builds on previous work by PNNL in the area of coupled deterministic-Monte Carlo simulations for complex radiation detection scenarios (Smith et al. 2008).

Each of the photon source terms (and instrument responses to those source terms) are modeled individually to create a library of high-precision (i.e., long MNCP runs to achieve high counting statistics) response functions that can then be quickly combined to support future parameter studies investigating variables such as enrichment level, U-232 concentration, and time since last separation. Examples from this response-function library and the "mixing" process used to predict the signatures from cylinders of various enrichments are given in Figure 5.

Previous AREVA NP measurements have been invaluable for benchmarking modeling results for $\mathrm{UF}_{6}$ cylinder neutron and gamma-ray signatures, and measured data will be used as much as possible during future phases of the ICVS modeling work. 

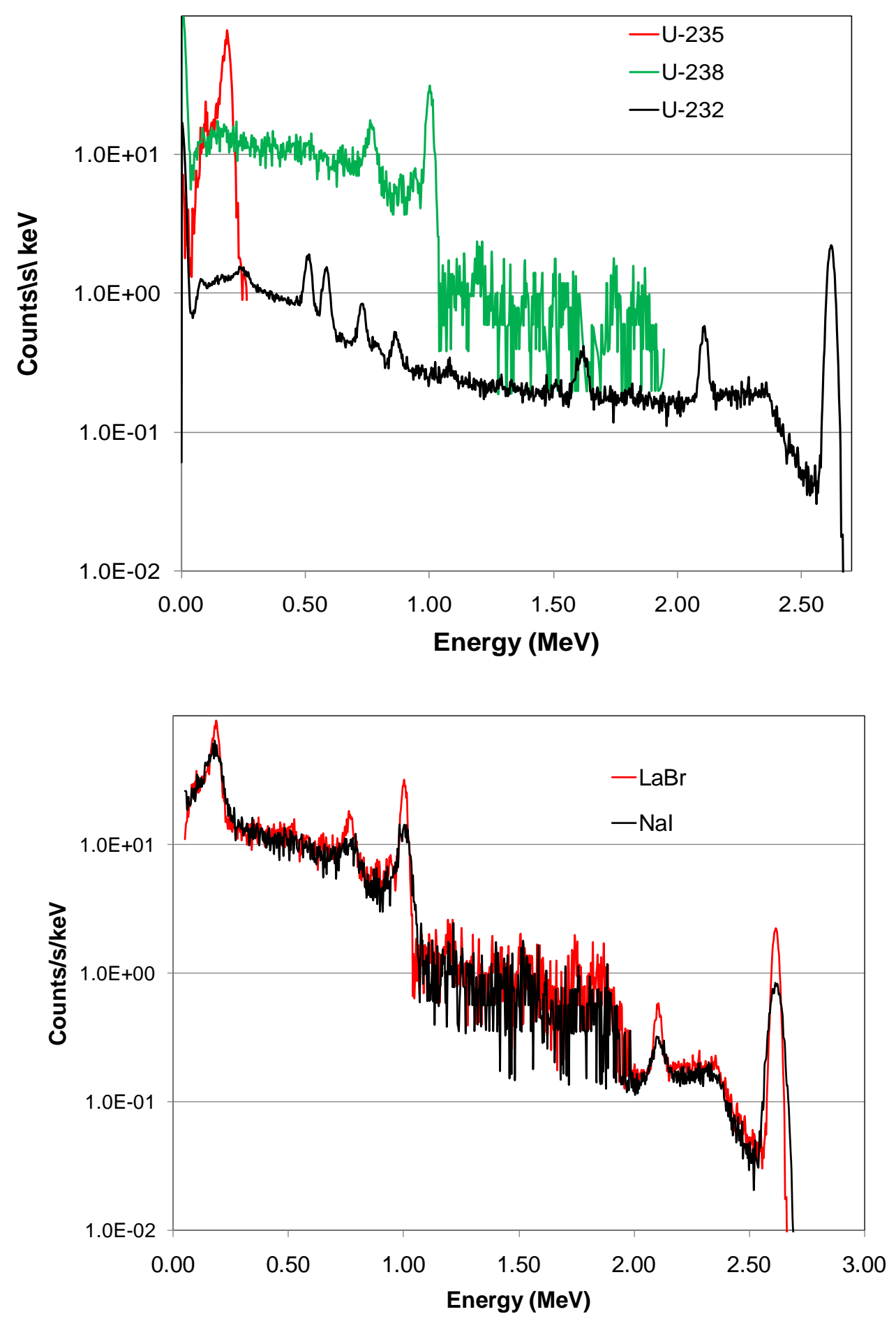

Figure 5. Examples of MCNP-modeled responses for the $7.5 \mathrm{~cm} \times 7.5 \mathrm{~cm} \mathrm{LaBr}_{3}(\mathrm{Ce})$ and $\mathrm{NaI}(\mathrm{Tl})$ spectrometers depicted in Figure 4. Top: Response functions for three major uranium isotopes (and daughters, assuming 6 months of build in) for $\mathrm{LaBr}_{3}(\mathrm{Ce})$. Bottom: Virtual cylinder responses (4.95\% enrichment), created by summing the isotope response functions in proper proportions, for both $\mathrm{LaBr}_{3}(\mathrm{Ce})$ and $\mathrm{NaI}(\mathrm{Tl})$. Note that the high-energy neutron-induced signatures (greater than $2.6 \mathrm{MeV}$ ) are not included in these plots. 


\subsection{Pulse-Processing Electronics}

PNNL's successful proof-of-principle study of non-traditional (neutron and neutron-induced highenergy gamma-ray) signatures for cylinder enrichment assay creates a new set of requirements for the pulse-processing electronics that are used to read out the $\mathrm{NaI}(\mathrm{Tl})$ or $\mathrm{LaBr}_{3}(\mathrm{Ce})$ gamma-ray spectrometers in the NDA prototype design. The most challenging requirement is a combination of analog-to-digital converter (ADC) resolution of at least 13 bits (8192 channels) and a high-voltage current supply of at least a few $\mathrm{mA}$. Individually, these specifications are easily achieved with commercially available systems but together, they are not, as described below. Note that this discussion is founded on a desire to use commercially available pulse-processing components to reduce the time and cost associated with the early incarnations of the NDA prototype system. Custom electronics may be considered in the future.

Standard NaI-based spectrometer systems use 1024 channels to span the energy range from 0 to 3,000 keV. This ensures that each channel is no more than a few $\mathrm{keV}$ wide, and that full-energy peak shapes (full-width at half maximum of $10 \mathrm{keV}$ or more) are smoothly represented because they are spanned by at least a few channels in the ADC (an important consideration for subsequent use of spectrum analysis algorithms). However, the non-traditional signatures being exploited by PNNL for cylinder assay extend to approximately $8,000 \mathrm{keV}$. Further, the $\mathrm{LaBr}_{3}(\mathrm{Ce})$ spectrometers that will be used in the NDA prototype have higher resolution (narrower peaks) than $\mathrm{NaI}(\mathrm{Tl})$. In combination, this results in the need for at least 8192 channels in the analog-to-digital conversion process (i.e., 13-bit ADC). Unfortunately, no standard commercial scintillator system provides such an ADC-pulse-processing electronics typically used with high-resolution HPGe spectrometers are required.

However, typical high-resolution HPGe pulse-processing systems cannot supply sufficient highvoltage current for scintillator photomultiplier tubes operating at high count rates (the reality in the cylinder assay scenario). Therefore, PNNL is using stand-alone high-voltage supplies appropriate for high count rates in medium-resolution scintillator systems.

PNNL procurements in this project included pulse-processing electronics to support preliminary testing of the $\mathrm{NaI}(\mathrm{Tl})$ and $\mathrm{LaBr}_{3}(\mathrm{Ce})$ spectrometers: high-rate photomultiplier tube bases, interface cabling to connect scintillator spectrometers to PNNL's existing HPGE ADC modules, and portable highvoltage power supplies. 


\subsection{Summary and Future Work}

An automated method for verifying U-235 enrichment in product cylinders would enable 100 percent verification, as opposed to today's sampling-based verification approach. Such automated verification, at a series of key measurement points, could also enable a mass-balance calculation for an enrichment plant in which all inputs and outputs of the facility — feed, tail, and product cylinders-are measured. Using more-penetrating neutron and high-energy gamma-ray signatures capable of interrogating the entire $\mathrm{UF}_{6}$ volume, rather than just a small volume near the outer cylinder wall, could decrease systematic uncertainties due to wall-thickness variations or variations in $\mathrm{UF}_{6}$ enrichment within a cylinder.

Proof-of-principle measurements (prior work under PNNL LDRD funding) of neutron and highenergy gamma-ray signatures, using moderated He-3 neutron detectors and large-volume NaI spectrometers in a fixed-geometry, portal-like configuration supported an early assessment for the viability of PNNL's ICVS concept. Analysis of a small product cylinder population indicated that the combination of non-traditional assay signatures (e.g., neutron-induced high-energy photons) with traditional signatures (i.e., $185-\mathrm{keV}$ line from U-235) has the potential to meet target uncertainties for enrichment verification in product cylinders for a range of feed materials and enrichment processes, and that these methods may be extensible to the larger tail and feed cylinders as well (although no measurements were performed on tail and feed cylinders).

The scope described in this report, supported by NA-243, builds on the previous PNNL LDRD study completed in mid-FY2009. A proposal has been submitted to NA-241 (specifically, the Next Generation Safeguards Initiative Technology Development portfolio) to fund a FY10-FY12 project to fully develop and demonstrate the ICVS concept, with FY10 activities concentrated on design, prototyping, and testing of the enrichment measurement component of the ICVS.

The three key tasks for this bridge project were: 1) complete a preliminary design of an NDA prototype system intended for FY10 measurements at an AREVA fuel fabrication facility and subsequent demonstration at an operating enrichment plant, 2) continue development of MCNP models that are critical to hardware design optimization and the development of enrichment analysis algorithms, and 3) purchase and perform initial testing of specific pulse-processing electronics to augment the existing instrumentation that will be used in the NDA prototype system. Each of these objectives was achieved between June and October 2009 under NA-243 funding, and the results of this work will directly feed the Technology Development project, should it be funded by NA-241 for FY10-FY12.

The three-year project proposal to NA-241 includes tasks to complete the final design and fabrication of the prototype NDA system, development and refinement of enrichment analysis algorithms that combine non-traditional signatures with an automated form of the traditional techniques, continued development of the modeling framework to support design studies and algorithm development, and field measurements and demonstrations. A summary of the proposal is provided as Appendix A to this report. 


\subsection{References}

Curtis MM, SL E and MW Shaver. 2009. UF6 Cylinder Verification: Portal Monitor Conceptual Design. PNNL-18171, Pacific Northwest National Laboratory, Richland, Washington.

Kuhn E, et al. 2001. International Target Values 2000 for Measurement Uncertainties in Safeguards Nuclear Materials. IAEA STR-327, International Atomic Energy Agency, Vienna, Austria.

Mosher SW, TM Miller, TM Evans and JC Wagner. 2009. “Automated Weight-Window Generation for Threat Detection Applications Using ADVANTG.” In Proceedings of the 2009 International Conference on Advances in Mathematics, Computational Methods, and Reactor Physics (M\&C 2009). May 3-7, 2009, Saratoga Springs, New York. American Nuclear Society, LaGrange Park, Illinois. On CD-ROM.

Smith LE, MM Curtis, MW Shaver, J Benz, A Misner, E Mace, DV Jordan, D Noss and H Ford. 2009. “Development of a Portal Monitor for UF6 Cylinder Verification.” In 2009 Institute of Nuclear Materials Management Conference. July 12-16, 2009, Tucson, Arizona. On CD-ROM, Institute of Nuclear Materials Management, Deerfield, Illinois.

Smith LE, JE Ellis, CJ Gesh, J R J McConn, GH Meriwether, EA Miller, RT Pagh, AB Valsan and TA Wareing. 2008. "Deterministic Transport Methods for the Simulation of Gamma-Ray Spectroscopy Scenarios.” IEEE Transactions on Nuclear Science 55(5):2598-2606. 



\title{
Appendix A
}

\section{Summary of FY10-12 Proposal}

Title: An Integrated Cylinder Verification Station for Enrichment Plant Safeguards

\begin{abstract}
Current $\mathrm{UF}_{6}$ cylinder enrichment measurements performed by the IAEA are man-power intensive and often inaccurate due to wall-thickness variations and heterogeneity of enrichment over the cylinder volume. PNNL proposes an automated $\mathrm{UF}_{6}$ cylinder verification station to provide $100 \%$ cylinder verification and full-cylinder-volume enrichment assay, as well as the integration of IAEAapproved load cells, surveillance cameras, positive cylinder identification and authenticated data storage until operator arrival.
\end{abstract}

\section{Scope}

This project will build on a strong foundation of $\mathrm{UF}_{6}$ enrichment assay instrumentation and methods development funded by PNNL internal investments in FY08-FY09, and NA-24 investments in the testing and characterization of ancillary systems (e.g., load cell and cylinder-identification technologies) during that same time period. Key tasks in the proposed work are summarized below.

\section{Task 1: Optimize Radiation Detection Instrumentation}

Previous cylinder measurements at an AREVA fuel fabrication facility in Richland, WA and an accompanying modeling effort have highlighted a number of key design features to incorporate when building a demonstration prototype (e.g., specific collimation design, high-rate digital pulse-processing electronics). PNNL will develop a prototype system appropriate for additional measurements at AREVA and a field demonstration at an operating enrichment plant. The prototype will include both $\mathrm{NaI}(\mathrm{Tl})$ and $\mathrm{LaBr}_{3}(\mathrm{Ce})$ gamma-ray spectrometers (to allow head-to-head comparison of efficacy for cylinder assay in a fixed geometry portal) and a He-3 neutron detection module.

\section{Task 2: Develop and Optimize Enrichment Analysis Algorithms}

Previous PNNL work provided proof-of-principle data to support the concept of collecting morepenetrating neutron-related signatures and enabling full-volume cylinder enrichment verification, in addition to the localized 185-keV-based assay utilized today. Because these more-penetrating neutron signatures derive from U-234, a stable U-234/U-235 ratio is needed in order to be a useful measure of U-235 enrichment. While preliminary data suggests this is a reasonable assumption for ore-based feed and a facility-specific calibration, further exploration of the U-234/U-235 ratio, across feed material types and enrichment processes, is needed to more fully understand the potential (and shortcomings) of neutron-based signatures. The proposed work would also include the development of an automated form of the traditional U-235 185-keV peak enrichment-meter calculation. Appropriate methods for combining 
the non-traditional, indirect (based on U-234 emissions) and traditional, direct (based on U-235 185-keV emissions) signatures will be developed, and the utility of other full-spectrum analysis techniques (e.g., wall-thickness estimation) will be explored and tested.

\section{Task 3: Refine Modeling Framework}

High-fidelity modeling of the $\mathrm{UF}_{6}$ cylinder assay scenario is a time- and resource-efficient complement to other development tasks. For example, the impact of different background shielding configurations can be quickly explored in simulation, but is time-consuming and expensive in the field. Simulation can also be used to generate large libraries of "virtual assay signatures" to support algorithm development, over the parameter space important to a viable instrument (e.g., enrichment levels, U-232 contamination levels and wall-thickness variation). PNNL developed a functional and preliminarily validated modeling framework in previous work; the proposed work will expand and refine this framework.

\section{Task 4: Field Measurements and Demonstration}

The AREVA fuel fabrication facility provides a convenient and cost-effective test bed for additional cylinder measurements, and those measurements will support the initial testing and evaluation of the NDA prototype system and accompanying algorithms. However, the AREVA facility cannot emulate the complex environment of an operating enrichment plant and consequently, PNNL is also seeking to perform field measurements at a domestic or international enrichment facility, perhaps in conjunction with IAEA's ongoing testing of their laser item identification system (L2IS) and/or the Next Generation Surveillance System (NGSS).

\section{Task 5: Integration Plans for Ancillary Systems}

Previous NA-24 funding support the purchase and testing of a Toledo-Mettler floor scale. In this project, methods for integrating the scale and the NGSS with the NDA systems will be defined and explored. The integration of IAEA's L2IS and a secure mailbox system will also be considered, in consultation with IAEA.

Tasks (Target completion dates)

Task 1: Optimize Radiation Detection Instrumentation (9/1/2010)

Task 2: Develop and Optimize Enrichment Analysis Methods (6/1/2011)

Task 3: Refine Modeling Framework (1/1/2011)

Task 4: Field Measurements and Demonstration (3/30/2012)

Task 5: Integration Plans for Ancillary Systems (8/1/2012)

\section{Deliverables}

9/30/2010: Technical report summarizing progress to date

9/30/2011: Technical report summarizing progress to date and AREVA measurement results

3/30/2012: Demonstration at operating enrichment plant

9/30/2012: Technical report summarizing comparative performance and findings 



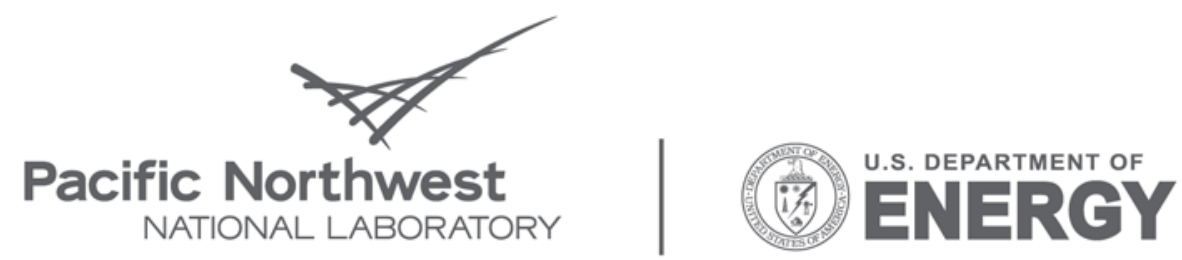

Proudly Operated by Battelle Since 1965

902 Battelle Boulevard

P.O. Box 999

Richland, WA 99352

1-888-375-PNNL (7665)

www.pnl.gov 